\title{
A new chapter in the problem of the reduction of chemistry to physics: The Quantum Theory of Atoms in Molecules
}

\author{
Jesus Alberto Jaimes Arriaga ${ }^{\dagger}$, Sebastian Fortin ${ }^{\dagger \dagger}$, Olimpia Lombardi ${ }^{\dagger}$
}

\begin{abstract}
The problem of the reduction of chemistry to physics has been traditionally addressed in terms of classical structural chemistry and standard quantum mechanics. In this work, we will study the problem from the perspective of the Quantum Theory of Atoms in Molecules (QTAIM), proposed by Richard Bader in the nineties. The purpose of this article is to unveil the role of QTAIM in the inter-theoretical relations between chemistry and physics. We argue that, although the QTAIM solves two relevant obstacles to reduction by providing a rigorous definition of chemical bond and of atoms in a molecule, it appeals to concepts that are unacceptable in the quantum-mechanical context. Therefore, the QTAIM fails to provide the desired reduction. On the other hand, we will show that the QTAIM is more similar to Bohmian mechanics and that the basic elements of both theories are closely related.
\end{abstract}

\section{Introduction}

Although the philosophy of chemistry has experienced a strong development during the last decades, the problem of the relation between chemistry and physics is still in the spotlight, since it affects the theoretical independence of chemistry as a scientific discipline. In this context, the most serious obstacles arise when quantum mechanics attempts to account for molecular structure. It is precisely in this regard that the Quantum Theory of Atoms in Molecules (QTAIM), proposed by the Canadian quantum chemist Richard Bader (1990), has begun to attract the attention of chemists and philosophers of chemistry (see, e.g., Matta et al. 2011; Matta 2013). In fact, the QTAIM is introduced as the theory that supplies the rigorous theoretical foundation, based on quantum mechanics, to the chemical notion of molecular structure.

Bader proposes the QTAIM as an extension of quantum mechanics for the study of chemical systems, and not as an alternative strategy to obtain information from the wave function. On this basis, in the framework of the QTAIM, molecular structure is defined in terms of the electronic

\footnotetext{
${ }^{\dagger}$ CONICET - Universidad de Buenos Aires.

${ }^{\dagger}$ CONICET - FCEN, Universidad de Buenos Aires. Corresponding author: sfortin@ conicet.gov.ar
} 
density, and certain tools of classical mechanics are used to account for the notion of chemical bond. In this way, it is supposed that the theory supplies a reductionist framework that lays the foundations for a unifying physical theory of molecular structure. However, much of the language used by the QTAIM presupposes classical concepts that are completely alien to the conceptual world of standard quantum mechanics (SQM).

On the other hand, it is interesting to notice that the problem of the relations between chemistry and physics has been almost always addressed from the perspective of SQM, forgetting other formalisms as that of Bohmian quantum mechanics (BQM). But when the QTAIM is considered, it is natural to suspect that its conceptual scheme has more affinity to BQM than to SQM. For this reason, the analysis of the relationships of the QTAIM both with SQM and with BQM is a task that deserves to be undertaken.

The aim of this article consists in unveiling the role played by the QTAIM in the theoretical relationships between chemistry and physics. We will argue that the QTAIM is closer to BQM than to SQM. With this purpose, the article is organized as follows. Section 2 will be devoted to provide a brief presentation of the QTAIM, following the lead of its main concepts. In Section 3, the main foundational problems of SQM will be introduced. In Section 4, BQM will be explained, emphasizing its conceptual basis. In the light of the previous material, Section 5 will be devoted to discuss the similarities and differences between the QTAIM and each one of the other two theories. Finally, in the Conclusions, the main results obtained in the article will be recalled, stressing the philosophical aspects that are still in need of further treatment.

\section{The main tenets of the QTAIM}

According to Bader, the manifestation of matter in real -physical- space is represented by the spatial distribution of the electronic density associated to the systems that inform matter, that is, atoms and molecules. From his perspective, the electronic density embodies all the physical information about matter, and its topology defines the concepts of atom, bonding, molecular structure and structural stability. It is for this reason that the author considers that a theory based on the electronic density may establish the link between the languages of chemistry and of physics (Bader 2011).

The first definition of electronic density was formulated by Erwin Schrödinger (1926b, 1926c), as a continuous distribution of electricity in the physical space. He stresses that the wave function $\Psi$ should not be directly used to interpret the physical phenomenon, since it is a function in configuration space and not in physical space. Instead, he advocates for using $\Psi$ only to obtain the quantity $\Psi^{*} \Psi$, since "the charge of the electron is not concentrated in a point, but is spread out through the whole space, proportional to the quantity $\Psi^{*} \Psi$ " (Schrödinger 1926c: 1066). Schrödinger is, then, interested in the electronic charge, $e \rho(r)$, 
where $e$ is the charge of the electron and $\rho(r)$ is the electronic density, and computes $\rho(r)$ by means of the following strategy. First, $\Psi^{*} \Psi$ is integrated over the coordinates of all except one electron, which is maintained at rest in position $r$, where one wants to know the electronic density. Then, the same integral is obtained for all the remaining electrons, considered at rest at the same position. Finally, all the integrals are added, in such a way that the following expression for $\rho(r)$ is obtained:

$$
\rho(r)=N \int \Psi^{*} \Psi d \tau
$$

If $N$ is the number of electrons in the system, then this density $\rho(r)$ represents, from the quantum point of view, the probability of finding electrons at each point in space. Additionally, it is necessary to introduce a correction in order to take into account the electronic spin (Bader 2010).

In the QTAIM (Bader 1990, 1991), molecular structure is characterized by the topology of the electronic density $\rho(r)$ : the morphology of such a topology is what defines the atoms in molecules. This morphology is given by the number and types of the topological features of $\rho(r)$, which can be determined by studying the mathematical properties of the density gradient field $\nabla \rho(r)$. The points where $\nabla \rho(r)=0$ are called critical points. Critical points can be either local maxima, local minima and saddle points of the electronic density, depending on the value of the derivative of the gradient field $\nabla \rho(r)$. On this basis, the positions of the nuclei correspond to the local maxima of $\rho(r)$, and this topological feature leads to the natural partition of the molecular space into mononuclear regions, which, in the QTAIM, are identified with the atoms in the molecules. The surface that limits an atom in a molecule is a separatrice surface, that is, a surface of zero-flux of the density gradient field $\nabla \rho(r)$ : this is interpreted as the fact that the electronic density of the region limited by that region -the atom- is constant in time. According to Bader, the association of these topological features with the elements of molecular structure lays the foundations of a rigorous theory of chemical structure.

In this new theoretical framework, the chemical concept of chemical bond is replaced by that of bond path since, as Bader argues, the concept of chemical bond is insufficient to account for the totality of models of bond employed in chemistry. Likewise, the author points out that the concept of bond path is a universal indicator that two atoms are bonded, and thus provides a unified physical vision for all bonding interactions used by chemists (Bader 2009, 2011). In the author's words:

"The reductionist approach afforded by QTAIM offers a clear solution to the myriad of personal views and models of bonding. As has been amply demonstrated by appeal to physics, the presence of a bond path linking a pair of atoms fulfills the sufficient and necessary conditions that the atoms are bonded to one another. This definition, which necessarily applies to quantum mechanical 
densities, transcends all bonding schemes and categories and provides a unified physical understanding of atomic interactions. One assumes such unification to be a primary goal of any physical theory." (Bader 2011: 20).

Moreover, the notion of bond path is rooted in a measurable quantity, namely, the electron density; as a consequence, it is possible to define a bond path operator as a Dirac observable, and so to obtain the expected values corresponding to this property. In this case, it can be proved that, when two atoms interact with each other, they are linked by means of an accumulation of electron density between them (Bader 2009, 2011), a fact already observed in the first developments of quantum chemistry (Heitler and London 1926). It is also possible to assign a certain electron density to each atom in the molecule, which determines the contribution of that atom to the properties of the whole system, the molecule (Bader 2007).

Summing up, the QTAIM offers a reductionist scheme in which quantum bonding is interpreted in terms of the theorems of quantum mechanics, an idea that had been proposed by John Clarke Slater in 1933. From this perspective, the idea that a molecule is a collection of atoms can be retained: all atoms contribute to the properties to the molecule. In this way, the QTAIM also provides a physical foundation for the concept of functional group.

\section{Standard quantum mechanics}

The standard version of quantum mechanics was developed by the founding fathers of the theory, such as Niels Bohr, Werner Heisenberg, Max Born and Erwin Schrödinger, among others, in an attempt of understanding the atomic world through the new concepts. Nevertheless, the quantum view stood completely against the foundations of classical physics. It came along with principles so anti-intuitive that physicists of the level of Einstein and Schrödinger always maintained a position contrary to the new theory, supposing that quantum mechanics was still incomplete and that a better description of the world was possible (Bricmont 2016).

In 1926, Schrödinger published a series of articles where he developed the principles of a new theory that intended to give an account of the behavior of the particles that constitute matter. A year before, Heisenberg had proposed a theory based on matrices and non-commuting properties with the same purpose. Nevertheless, later it was proved that both theories were equivalent. The dynamical equation of the theory, formulated by Schrödinger, can be written as:

$$
i \hbar \frac{\partial \Psi(r, t)}{\partial t}=\left[-\frac{\hbar^{2}}{2 m} \bar{\nabla}^{2}+V(r, t)\right] \Psi(r, t)
$$

where $\hbar$ is Planck constant divided by $2 \pi, m$ is the mass of the particle, $\bar{\nabla}^{2}$ is the Laplacian operator, $V(r, t)$ is the potential energy of the system, and $\Psi(r, t)$ is the wave function that represents the state of the quantum system. In the usual formalism, the wave function is the 
representation in spatial coordinates of the state vector $|\Psi\rangle$ ("ket", in Dirac notation), which belongs to a Hilbert space $\mathcal{H}$, whose vectors have complex components; the result of the scalar product of two state vectors is a real number with physical meaning.

In turn, the measurable properties of a quantum system are called observables, which are represented by linear operators acting on the Hilbert space $\mathcal{H}$ (see, e.g. Ballentine 1998). For any observable $O$, a linear operator $\hat{O}$ is defined, which has its eigenvectors $\left|o_{i}\right\rangle$ and eigenvalues $o_{i}$, in such a way that when the operator is applied to its eigenvector, the same eigenvector multiplied by the corresponding eigenvector is obtained: $\hat{O}\left|o_{i}\right\rangle=o_{i}\left|o_{i}\right\rangle$. According to quantum mechanics, the eigenvalues $o_{i}$ associated to each operator $\hat{O}$ are the possible values of the observable $O$ obtained when the corresponding property is measured. In turn, the eigenvectors $\left|o_{i}\right\rangle$ of the operator $\hat{O}$ form a basis of the Hilbert space $\mathcal{H}$. With these operators, relevant physical magnitudes can be computed in the following way: the expectation value of the observable $O$, associated to the operator $\hat{O}$, in the quantum state $|\Psi\rangle$ is computed as:

$$
\langle\hat{O}\rangle_{|\Psi\rangle}=\langle\Psi|\hat{O}| \Psi\rangle
$$

Let us suppose that $\left\{\left|a_{i}\right\rangle\right\}$ is a basis of the Hilbert space $\mathcal{H}$. This means that any state vector $|\Psi\rangle$ of the quantum system can be expressed as a linear combination of the vectors of that basis:

$$
|\Psi\rangle=\sum_{i} \alpha_{i}\left|a_{i}\right\rangle
$$

where the coefficients $\alpha_{i}$ are complex numbers. But, since there are infinite basis of the Hilbert space, the same state vector can be expressed in terms of the vectors of another basis, say, $\left\{\left|b_{i}\right\rangle\right\}$ :

$$
|\Psi\rangle=\sum_{i} \beta_{i}\left|b_{i}\right\rangle
$$

Where, again, the coefficients $\beta_{i}$ are complex numbers, which keep a precise relation with the coefficients $\alpha_{i}$ corresponding to the decomposition of the vector in the previous basis.

This mathematical feature expresses one of the most striking principles of quantum mechanics, the principle of superposition, according to which any linear combination of possible quantum states of a system is also a possible quantum state of the system. As an example, let us suppose the observable spin of an electron, which has two possible values, spin-up $\uparrow$ and spin-down $\downarrow$, corresponding to the eigenvectors $|\uparrow\rangle$ and $|\downarrow\rangle$ of the operator $\hat{S}$. Therefore, $|\uparrow\rangle$ and $|\downarrow\rangle$ are two possible states of the electron, but also are any vector $|\varphi\rangle$ obtained as a linear combination of them: $|\varphi\rangle=c_{1}|\uparrow\rangle+c_{2}|\downarrow\rangle$. In this case, it is said that $|\varphi\rangle=c_{1}|\uparrow\rangle+c_{2}|\downarrow\rangle$ is a superposition of the states $|\uparrow\rangle$ and $|\downarrow\rangle$ corresponding to spin-up $\uparrow$ and spin-down $\downarrow$, respectively. It must be emphasized that this new state is neither spin-up 
nor spin-down, but a combination of both: this is very weird from a classical viewpoint, since classically any property has a definite value and superpositions of different values make no sense.

Another peculiar feature of quantum mechanics is Heisenberg's principle of uncertainty, which can be expressed as:

$$
\Delta q \Delta p \geq \hbar / 2
$$

From this principle, if the position of a quantum particle is known with complete precision, it is not possible to count with precise knowledge of its momentum (or velocity), and vice versa. This principle is consequence of the mathematical formalism, since incompatible observables exist, whose operators do not commute:

$$
[\hat{A}, \hat{B}]=\hat{A} \hat{B}-\hat{B} \hat{A} \neq 0
$$

In order to understand the meaning of this principle, let us consider a quantum system with an observable $A$ represented by an operator $\hat{A}$ with eigenstates $\left|a_{i}\right\rangle$ and a different observable $B$ represented by an operator $\hat{B}$ with eigenstates $\left|b_{i}\right\rangle$. As explained above, the eigenstates $\left|a_{i}\right\rangle$ can be expressed in terms of the basis $\left\{\left|b_{i}\right\rangle\right\}$ as:

$$
\left|a_{i}\right\rangle=\sum_{i j} \gamma_{i j}\left|b_{j}\right\rangle
$$

Since the two basis $\left\{\left|a_{i}\right\rangle\right\}$ and $\left\{\left|b_{i}\right\rangle\right\}$ are different, the coeficients $\gamma_{i j}$ cannot be adopt the values 0 or 1 . This means that, if the observable $A$ has a definite value, say $a_{k}$ corresponding to the eigenstate $\left|a_{k}\right\rangle$, the observable $B$ has no definite value. For this reason it is said that quantum mechanics is contextual, that is, only observables defining the same basis of he Hilbert space can have definite values simultaneously (see Bub 1997).

Up to this point, the quantum systems were referred to as if they were "individual objects"; nevertheless, in the light of certain aspects of the theory, it is not very clear that they can be treated as such. Traditionally, an individual object has some "principle of individuality" that distinguishes it from other objects and makes it the same through time; therefore, it can be unambiguously traced back over space and time. Quantum systems, such as electrons, protons and neutrons, on the contrary, cannot be considered individuals in that traditional sense. In fact, some properties of quantum systems, like position and velocity, are not always well-defined due to the contextuality of the theory; this feature prevents them to be endowed with a precise trajectory over space and time. On the other hand, the requirement of symmetrization (bosons) or anti-symmetrization (fermions) gives support to an interpretation of quantum entities as non-individuals (French and Krause 2006). This lack of individuality is not a merely epistemic limitation, but an ontological feature with deeper implications. Considering the quantum indistinguishability, some authors have developed a reformulation of quantum mechanics based on quasisets, in which indistinguishability is taken as a primitive 
notion (Domenech et al. 2008 and 2010). Finally, when two independent quantum systems interact, they cannot be individualized in the composite system anymore, since they constitute a new system, not analyzable in the original components (Ballentine 1998). This is the case of chemical molecules, which, from the viewpoint of SQM must be treated in an holistic and non-local way (Primas 1983).

\section{Bohmian quantum mechanics}

In spite of the great success of SQM, due to its conceptual puzzles, an alternative theory was developed by David Bohm in the early 50s (Bohm 1952a, 1952b). This theory appeals to hidden variables to give a complete description of quantum phenomena. In this way, it offers a description that is closer to the principles of classical physics, since the trajectories of the subatomic particles can be defined. It is interesting to stress that, in spite of the deep ontological differences between SQM and BQM, both theories are empirically equivalent, that is, they lead to the same predictions at he experimental level.

In order to understand the main tenets of BQM, it is convenient to briefly recall the principles of the Hamilton-Jacobi formulation of classical mechanics, according to which the motion of a physical object is obtained from the following equation:

$$
\frac{\partial S}{\partial t}+\frac{(\bar{\nabla} S)^{2}}{2 m}+V=0
$$

where $S$ is the action and $V$ is the potential. On the basis of this formalism, the momentum of the object can be computed as follows (Goldstein et al. 2002):

$$
\bar{p}=\bar{\nabla} S
$$

In BQM, the motion of particles in the real space of three dimensions is ruled by the following equation:

$$
\frac{d Q_{i}}{d t}=\frac{\hbar}{m_{i}} \operatorname{Im} \frac{\nabla_{i} \Psi\left(Q_{1}(t), \ldots, Q_{N}(t), t\right)}{\Psi\left(Q_{1}(t), \ldots, Q_{N}(t), t\right)}
$$

where Im indicates the imaginary part of the magnitude, $Q_{i}(t)$ and $m_{i}$ represent the position in three-dimensional space and the mass of the $i$-particle, respectively, and $\Psi$ is the wave function, that satisfies eq. (2). (see Tumulka 2004). The motion equation (11) is the result of introducing a new force, which depends on all the particles of the universe. In order to understand the source of that force, let us begin by the Schrödinger equation (eq. (2)), where the wave function $\Psi$ is a complex function; so it can be written as follows (see Holland 1993): 


$$
\Psi=R e^{\frac{i S}{\hbar}}
$$

where $R$ is the modulus of the wave function, such that $R^{2}=P$ is the density of probability, and $S$ is the phase. When eq. (10) is introduced into eq. (2), and a change of variables $R \rightarrow P$ is performed, the following equations are obtained:

$$
\begin{gathered}
\frac{\partial P}{\partial t}+\bar{\nabla} S\left(P \frac{\nabla S}{m}\right)=0 \\
\frac{\partial S}{\partial t}+\frac{(\bar{\nabla} S)^{2}}{2 m}+V-\frac{\hbar}{4 m\left(\frac{\bar{\nabla} P}{P}-\frac{1}{2} \frac{(\bar{\nabla} P)^{2}}{P^{2}}\right)}=0
\end{gathered}
$$

Where eq. (13) is a continuity equation for $P$, and the new force acting on the particles results from a quantum potential, given by:

$$
U=\frac{\hbar}{4 m\left(\frac{\bar{\nabla} P}{P}-\frac{1}{2} \frac{(\bar{\nabla} P)^{2}}{P^{2}}\right)}
$$

In this way, considering the total potential as $V_{\text {tot }}=V+U$, eq. (14) can be written as:

$$
\frac{\partial S}{\partial t}+\frac{(\bar{\nabla} S)^{2}}{2 m}+V_{t o t}=0
$$

which is an analogous equation to the Hamilton-Jacobi equation (eq. (9)), in such a way that the momentum can be still computed by means of eq. (10), and the trajectories can be obtained with eq. (16). The limitation to effectively compute the particles' trajectories is that the initial conditions of the quantum system are unknown. For this reason, Bohm appeals to the statistical reading of the wave function, and considers that the initial conditions are distributed with a density of probability $P=|\Psi|^{2}$. Nevertheless, Bohm stresses that this probability distribution is not due to the conceptual nature of the quantum systems, but it is "merely a consequence of our ignorance of the precise initial conditions of the particle" (Bohm 1952a: 171).

Although BQM was applied to successfully describe many physical situations, here we will focus on its application to chemical systems; in particular, we will study the hydrogen molecule. With this purpose, let us begin by considering the hydrogen atom; the $1 s$ state of the electron has the following form:

$$
\Psi=f(r) e^{-\frac{i E t}{\hbar}}
$$

where $E$ represents the energy. So, the phase function is $S=E t$ and, according to eq. (10), momentum is given by $\bar{p}=\bar{\nabla} S$, which means that the electron is at rest. However, the 
position of the electron cannot be known precisely, since the initial conditions are unknown and were defined statistically. Nevertheless, the result agrees with classical physics: since the electron does not move around the nucleus, it does not lose its energy and does not collapse with the nucleus, a result predicted by electromagnetism. What can nevertheless be computed with precision is the distance between the electron and the nucleus. In fact, the energy is given by:

$$
E=V+U
$$

Where the quantum potential $U$ must be opposed to the potential energy $V$ and, as a consequence, tends to equilibrate it. When the energy $E$ corresponds to that of the fundamental state, the distance between electron and nucleus is equal to the Bohr radius. Regarding the remaining orbitals, the phase is not zero, $S \neq 0$; in this case, the radius can be computed as:

$$
r=r_{0} \sin \theta_{0} \quad \theta=\theta_{0} \quad \phi=\phi_{0}+\frac{m \hbar t}{m_{0} r_{0}^{2} \sin ^{2} \theta_{0}}
$$

where $r, \theta, \phi$ are the polar coordinates, $r_{0}, \theta_{0}, \phi_{0}$ are the initial polar coordinates, $m$ is the azimuthal quantum number, and $m_{0}$ is the mass of the electron. Eqs. (19) are interpreted as describing an electron that orbits around the nucleus with a constant radius $r=r_{0} \sin \theta_{0}$ and an angular velocity proportional to $\hbar /\left(m_{0} r_{0}^{2} \sin ^{2} \theta_{0}\right)$ (Holland 1993: 150). However, the orbit of the electron is different from Bohr orbit, since in the Bohmian case the orbit is not in the equatorial plane, even if it is parallel to it. Moreover, quantization does not correspond to the orbit, but to the velocity of the particle.

This preliminary study supplies the basis to describe the hydrogen molecule in the context of the BQM. Let us recall that, in chemistry, it is said that two atoms are bonded when they share electrons; in the particular case of the hydrogen molecule $\mathrm{H}_{2}$, the two atoms are linked by a covalent bond. Problems arise when quantum mechanics must account for the notion of chemical bond.

Given two hydrogen atoms in the state $1 s$ and far from each other, as explained above, their electrons are at rest. But when the two atoms get closer to form the molecule, the Schrödinger equation independent of time reads:

$$
\left[-\left(\frac{\hbar^{2}}{2 m}\right)\left(\nabla_{1}^{2}+\nabla_{2}^{2}\right)-\left(\frac{\hbar^{2}}{2 M}\right)\left(\nabla_{A}^{2}+\nabla_{B}^{2}\right)+V\right] \Psi=E \Psi
$$

where $\Psi=\Psi\left(\bar{x}_{1}, \bar{x}_{2}, \bar{r}_{A}, \bar{r}_{B}\right), \bar{x}_{i}(i=1,2)$ are the coordinates of the electrons, $\bar{r}_{j}(j=A, B)$ are the coordinates of the nuclei, $m$ is the mass of the electron and $M$ is the mass of the nucleus. Moreover, the potential energy is defined as:

$$
V=-\frac{e^{2}}{r_{A 1}}-\frac{e^{2}}{r_{B 1}}-\frac{e^{2}}{r_{A 2}}-\frac{e^{2}}{r_{B 2}}+\frac{e^{2}}{r_{12}}+\frac{e^{2}}{r_{A B}}
$$


where $r_{\alpha \beta}$ are the distances between the particles $\alpha$ and $\beta$. Eq. (20) can be solved by means of conventional methods, obtaining two possible solutions, corresponding to the bonding and antibonding wave function, and whose associated energy is given by:

$$
E_{ \pm}=V+U_{ \pm}
$$

This result shows that the quantum potential $U_{ \pm}$is the factor responsible of the interaction between the electrons, which finally leads to the chemical bond. Additionally, from eq. (20) it can be concluded that the net force acting on the electrons is zero; this fact means that the electrons are at rest and, by considering the density of probability, it can be inferred that the most probable region to find the electrons is the region between the nuclei. This is a very surprising result, since the $\mathrm{BQM}$ seems to recover the notion of chemical bond proposed by Lewis before the consolidation of quantum mechanics.

\section{The QTAIM between the standard and the Bohmian views}

In the beginnings of quantum mechanics, Schrödinger attempted to attach a physical meaning to the wave function, proposing that it represented a kind of process of vibration in the atom (Schrödinger 1926a); according to the author, this view was more plausible than that according to which orbitals contain electrons. However, later, by contrast with his original ideas, he recognized that the wave function must not be applied directly to the interpretation of the physical phenomenon, but has to be only used to obtain the quantity $\Psi^{*} \Psi$, which represents the density of charge, by contrast to the interpretation of Born, who defined the same quantity, now designed as $|\Psi|^{2}$, as a density of probability.

According to Bader, Born's statistical interpretation of the wave function, finally incorporated to the SQM, is not consistent with the experiments of $\mathrm{X}$ rays used to measure the electronic density in crystals (Bader 2011); the author considers that this empirical fact supports the density of charge interpretation proposed by Schrödinger. In this sense, the QTAIM rejects the SQM's view of the wave function: the quantity $\Psi^{*} \Psi$ does not represent a probability; it is an intermediate quantity from which the concept of electronic density $\rho(r)$ can be defined. It is this concept that has physical meaning, since it determines the form of matter in real space (Bader 2011). In a certain sense, this rejection of the standard statistical interpretation is shared by the BQM, which discards the idea of an ontological probability associated to the wave function. From the Bohmian perspective, $\Psi$ has a clear physical meaning, since it is the mathematical representation of a real and objective field ruled by the Schrödinger equation (Bohm 1952a: 170). This real field exerts a force on the electron, in a similar way to the way how the electromagnetic field acts on an electric charge. 
On the other hand, the fact that the QTAIM adheres to an interpretation of the wave function different from that of the SQM excludes immediately several problems derived from the standard view. For example, the questions related with the principle of superposition vanish in the QTAIM, since in this theoretical context the concept of quantum state plays no role. The molecule's properties described by the QTAIM are always measurable quantities, represented by quantum observables; in particular, the electronic density $\rho(r)$ must not be conceived as a kind of state, but it is associated to an operator of the kind of Dirac's observables. Furthermore, the electronic density is the most important measurable property of the quantum system, because it encodes the concepts related to the molecular structure. In fact, it is in terms of the electronic density that the boundary condition of zero flux of an open system (an atom in a molecule) is defined, and the notion of bond path (describing bonding between atoms) can be characterized.

When the QTAIM is compared with the BQM regarding the main characters of the two theories, it may seem that they are very different. In fact, it is true that the BQM talks about the well defined trajectories of the particles, whereas the QTAIM considers the features of the electronic density to define the zero flux surfaces and the bonding paths. It is also true that the Bohmian trajectories are unobservable in principle, whereas the electronic density and its features are measurable properties of the molecule. Nevertheless, it is very natural to find a physically rational explanation of the concepts proposed by the QTAIM in the underlying dynamics of the Bohmian particles. For instance, the zero flux surfaces are the spatial boundaries of the atoms in the molecule precisely because no Bohmian particle can cross them; therefore, the Bohmian electrons remain confined to the basin of their respective nuclei and, for this reason, it can be said that each particular electron belongs to a particular atom. On the other hand, the QTAIM allows to construct the molecular graphs of the chemical systems, and these representations recover the classical idea of molecular structure in spatial terms, where two bonded atoms are linked by means of an accumulation of electronic density, in a way similar to the explanation given by the BQM.

Another aspect to consider is the individuality of the systems studied by the considered theories. In SQM, the Fermi-Dirac statistics for fermions and the Bose-Einstein statistics for bosons, due to quantum indistinguishability, seem to point to entities without individuality, since their permutation does not count as a different complexion; moreover, when two quantum systems form a composite system, they cannot be reidentified in the new whole. For these reasons, several authors consider that quantum systems are non-individuals in the sense that they have no individuality that makes them different from each other and that allows them to be reidentified along time. In the case of the BQM, by contrast, the quantum statistics do not originate in the indistinguishability of the quantum particles, but to the features of the quantum field: ontologically, Bohmian particles are classical individual particles, which 
describe precise trajectories and are clearly different from each other. In the case of the QTAIM, in turn, the atoms in the molecule are defined by the zero flux surface, which guarantees that the electronic density is time constant in each atom. This means that the atoms that form a molecule are individuals in the classical sense, since each one of them can be identified in the molecule and preserve their individuality through time since defined by their time-constant electronic density.

A final point to stress is the fact that Bader proposes to use certain theorems of quantum mechanics to interpret chemical bonding (Bader 2011b), a proposal already suggested by Slater in 1933. But, in doing so, the QTAIM introduces the concept of force, like Feynman's force or Ehrenfest's force, which, through the virial theorem, can be related with the kinetic and the potential energies of the system (Bader 2011b). But this language is dissonant with the SQM, by contrast to the BQM, in whose context seems to find a more comfortable place, since the Bohmian viewpoint supplies a more classical conception of quantum systems.

\section{Conclusions}

In the present paper we have treated the problem of the reduction of molecular chemistry to quantum mechanics from the perspective of the QTAIM. The starting point of this theory is the fact that the electronic density contains all the physical information about matter and, therefore, it is through the topological features of such a density that the concepts related with molecular structure and chemical bonding can be defined. Whereas traditional applications of quantum mechanics to chemical systems tend to dissolve the identity of the atoms in the molecule in favor of a holistic view, the aim of the QTAIM is to recover, with the language of quantum mechanics, the classical picture of the molecule as composed by individual atoms that is at the basis of structural molecular chemistry.

We have argued that QTAIM's view is not compatible with SQM, according to which the wave function is the state of the quantum system. Moreover, in SQM, quantum systems are not classical individuals that can be reidentified in the composite systems and through time. By contrast, QTAIM's aim of recovering the classical picture of the molecule seems to be closer to BQM's spirit, guided by the idea of overcoming the interpretive problems of quantum mechanics by coming back to an ontology of classical particles. For this reason, an underlying dynamics of Bohmian particles seems to be a good candidate to explain the QTAIM's proposal as an effective result. But a full argumentation in favor of this conclusion is beyond the limits of the present article and will be the subject matter of a future work. 
Acknowledgements: This publication was made possible through the support of grant 57919 from the John Templeton Foundation, and grant PICT-2812 of the Agencia Nacional de Promoción Cientítica y Tecnológica of Argentina.

\section{References}

Bader, R. (1990). Atoms in Molecules. A Quantum Theory. Oxford: Oxford University Press.

Bader, R. (1991). "A quantum theory of molecular structure and its applications". Chemical Review, 91: 893-928.

Bader, R. (2007). "Everyman's derivation of the theory of atoms in molecules". Journal of Physical Chemistry A, 111: 7966-7972.

Bader, R. (2009). "Bond paths are not chemical bonds". Journal of Physical Chemistry A, 113: $10391-10396$.

Bader, R. (2010). “The density in density functional theory”. Journal of Molecular Structure, 943: 2-18.

Bader, R. (2011). "On the non-existence of parallel universes in chemistry". Foundations of Chemistry, 13: 11-37.

Ballentine, L. (1998). Quantum Mechanics: A Modern Approach. Singapore: World Scientific Publishing Company.

Bricmont, J. (2016). Making Sense of Quantum Mechanics. Dordrecht: Springer.

Bohm, D. (1952a). "A suggested interpretation of the quantum theory in terms of hidden variables. I". Physical Review, 85: 166-179.

Bohm, D. (1952b). "A suggested interpretation of the quantum theory in terms of hidden variables. II". Physical Review, 85: 180-193.

Bub, J. (1997). Interpreting the Quantum World. Cambridge: University Press.

G. Domenech, F. Holik and D. Krause (2008). "Q-spaces and the foundations of quantum mechanics". Foundations of Physics, 38: 969-994.

G. Domenech, F. Holik, L. Kniznik and D. Krause (2010). "No labeling quantum mechanics of indiscernible particles", International Journal of Theoretical Physics, 49: 3085-3091.

French, S. and Krause, D. (2006). Identity in Physics: A Historical, Philosophical and Formal Analysis. Oxford: Oxford University Press.

Goldstein, H., Poole, C. P., and Safko, J. L. (2002). Classical Mechanics (3rd ed.). San Francisco: Addison Wesley. 
Heitler, W. and London, F. (1927). "Interaction between neutral atoms and homopolar binding according to quantum mechanics". Zeitschrit für Physik, 44: 455-472.

Holland, P. (1993). Quantum Theory of Motion. Cambridge: Cambridge University Press.

Matta, C. F. (2013). "Special issue: Philosophical aspects and implications of the quantum theory of atoms in molecules (QTAIM)". Foundations of Chemistry, 15: 245-251.

Matta, C. F., Massa, L., and Keith, T. A. (2011). "Richard F. W. Bader: a true Pioneer". Journal of Physical Chemistry A, 115: 12427-12431.

Primas, H. (1983). Chemistry, Quantum Mechanics and Reductionism. Berlin: Springer.

Schrödinger, E. (1926a). "Quatisierung als Eigenwertproblem (Erste mitteilung)". Annalen der Physik, 79: 361-376. Traducción al inglés en E. Schrödinger, Collected Papers on Wave Mechanics 1928. London and Glasgow: Blackie \& Son Limited.

Schrödinger, E. (1926b). "Quatisierung als Eigenwertproblem (Vierte mitteilung)". Annalen der Physik, 81: 437-490. Traducción al inglés en E. Schrödinger, Collected Papers on Wave Mechanics 1928. London and Glasgow: Blackie \& Son Limited.

Schrödinger, E. (1926c). "An undulatory theory of the mechanics of atoms and molecules". Physical Review, 28: 1049-1070.

Slater, J. C. (1933). "The virial and molecular structure”. Journal of Chemical Physics, 1: 687-691.

Tumulka, R. (2004). "Understanding Bohmian Mechanics: A Dialogue”. American Journal of Physics, 72: 1220-1226. 